\title{
La participación política de las comunidades migrantes en España. Nuevos y viejos dilemas
}

\section{Antía Pérez-Caramés}

Universidade da Coruña. Equipo de Sociología de las Migraciones Internacionales (ESOMI) antia.perez@udc.es

\section{Resumen}

Este texto constituye una presentación del monográfico «La participación política de las comunidades migrantes en España: Nuevos y viejos dilemas». Junto con un encuadre teórico que recoge las principales contribuciones más recientes al debate en torno a la participación política de las comunidades migrantes, se introducen los principales aspectos de diseño teórico y metodológico del proyecto de investigación sobre asociacionismo migrante en Galicia, que está en el origen de esta publicación, y se presenta brevemente cada uno de los artículos que compone el volumen.

Palabras clave: participación política; asociacionismo; inmigración; España; transnacionalismo

\section{Abstract. Political involvement of migrant communities in Spain: New and old dilemmas}

This text is an introduction to the case study "Political Involvement of Migrant Communities in Spain. New and Old Dilemmas." In addition to a theoretical framework that gathers the most recent major contributions to the discussion on the political involvement of migrant communities, the main aspects of the theoretical and methodological design for the research project on migrant associationalism in Galicia are introduced, as this project is at the origin of this publication. Each of the articles that comprise the study is briefly introduced.

Keywords: political involvement; associationalism; immigration; Spain; transnationalism 


\section{Sumario}

1. Introducción

2. La integración política de las comunidades migrantes: Entre el análisis sociodemográfico y la emergencia de la perspectiva transnacional
3. Estructura del monográfico

Agradecimientos

Referencias bibliográficas

\section{Introducción}

Este monográfico acerca siete contribuciones en forma de artículos de investigación que abordan los diversos interrogantes que se han abierto en los últimos años en los estudios sobre participación política de las comunidades migrantes, en un contexto que está también profundamente marcado por la recesión económica y la respuesta austeritaria a esta crisis. La transformación de las formas asociativas en el contexto de cambio de ciclo migratorio en España, la participación de las mujeres migrantes y el liderazgo femenino en las asociaciones, los enfoques del capital social y de la estructura de oportunidades políticas en el análisis del activismo político transnacional, la potencia de las asociaciones migrantes como agentes de codesarrollo y la representación política de los migrantes en dos países del boom migratorio como España e Italia son, de manera muy sintética, las temáticas abordadas por cada una de las contribuciones que componen este monográfico. A la variedad y actualidad temática de los textos que aquí se reúnen se suma también su carácter multidisciplinario, al confluir aportaciones de la sociología, la antropología, la ciencia política y las ciencias de la educación. Todos los artículos que se presentan derivan de investigaciones en curso o recientemente finalizadas en torno a la participación política de las comunidades migrantes.

El monográfico se ha elaborado parcialmente a partir de los resultados obtenidos en el proyecto de investigación titulado Asociacionismo migrante en Galicia: Lazos transnacionales y codesarrollo desde una perspectiva de género (PR 815A 2014-23), financiado por la Xunta de Galicia y que se llevó a cabo entre los años 2014 y 2016. Aunque presentaremos aquí brevemente los objetivos y el diseño metodológico de dicho estudio, hemos de indicar que este número se compone de otras cuatro contribuciones no vinculadas a esta, si bien parten de investigaciones originales y versan sobre distintos aspectos y perspectivas en torno a la participación política de las comunidades migrantes, lo que enriquece el conjunto del monográfico.

El estudio fue realizado a cargo de un equipo investigador formado por personas de las universidades de Vigo, A Coruña y Tras-os-Montes y Alto Douro (Portugal), en colaboración con la ONGD Ecos do Sur, una entidad de apoyo a las personas migrantes con sede en A Coruña y rango autonómico que cuenta con más de 35 años de experiencia en el terreno.

Los objetivos principales de la investigación consistían en analizar el panorama asociativo migrante en Galicia, estableciendo una comparación entre un 
estudio previo realizado por una miembro del equipo en el año $2006^{1}$; estudiar el papel que estas entidades podían desempeñar en tanto que entidades facilitadoras de codesarrollo y de integración de las comunidades migrantes, y, por último, indagar en las formas de participación política de las mujeres migrantes, haciendo especial hincapié en los procesos de incorporación y liderazgo asociativo y en los factores que promueven o inhiben la involucración de dicho colectivo.

En relación con los objetivos propuestos, el diseño metodológico combinaba el análisis de datos empíricos con técnicas de carácter cualitativo, como las entrevistas semiestructuradas a migrantes que participaban en asociaciones y también a agentes clave.

En lo que respecta al análisis de datos empíricos, en primer lugar se realizó una explotación de la base de datos sobre asociaciones de migrantes en Galicia procedente de la investigación titulada Asociacionismo e integración social de las inmigrantes en Galicia, desarrollada en 2006. Con este punto de partida, se levantó, entre los años 2015 y 2016, un censo de asociaciones de inmigrantes que, para actualizar el realizado una década antes, tuvo en consideración la información procedente de otras tres fuentes registrales: el portal Galicia Aberta de la Secretaría General de Emigración (Xunta de Galicia), el registro general de asociaciones de la Xunta de Galicia y el listado de asociaciones elaborado en el marco de un programa de intervención social realizado por la ONGD Ecos do Sur, que tenía como objetivo principal el fortalecimiento del tejido asociativo inmigrante. El proceso de elaboración y definición de variables de clasificación para el censo de 2016 se expone con más detalle en el artículo de este monográfico que lleva por título «Nuevas culturas asociativas en un contexto de cambio social: Análisis de la evolución del tejido asociativo inmigrante en Galicia (2006-2016)».

Una vez obtenido el censo de asociaciones de migrantes en Galicia se realizó un cuestionario telefónico a casi la mitad de las asociaciones censadas (30 de 79), lo que permitió ahondar en el conocimiento de las dinámicas organizativas de estas entidades ${ }^{2}$.Un análisis más detallado de los resultados de esta encuesta se puede encontrar en el artículo titulado "Capital social y redes políticas de las asociaciones de inmigrantes en Galicia».

Este análisis inicial permitió contar con un panorama actualizado del perfil y de la tipología de las asociaciones de migrantes en Galicia, lo que sirvió de base para la segunda fase de investigación, consistente en la realización del trabajo de campo cualitativo mediante entrevistas semiestructuradas a integrantes de 25 entidades de diverso perfil nacional y distribuidas por todo el territo-

1. Se trata del proyecto de investigación que llevaba por título Asociacionismo e integración social de las inmigrantes en Galicia, financiado por el Servicio Gallego de Igualdad (Xunta de Galicia) y dirigido por Iria Vázquez Silva.

2. Para la elaboración del cuestionario nos hemos servido, parcialmente y con las adaptaciones necesarias, del empleado en la investigación Participación política e integración social en Madrid: Asociaciones, activistas e inmigrantes (2003-2004), por lo que agradecemos a Laura Morales Díez de Ulzurrun su amable colaboración a este respecto. 
rio gallego. A estas entrevistas se suman otras 18 realizadas a agentes clave, representantes de entidades sin ánimo de lucro del tercer sector de apoyo a la población migrante y actores institucionales de los niveles autonómico y local de la Administración. El guión de las entrevistas a miembros de asociaciones migrantes abordaba temas como la trayectoria migratoria y de participación política de la persona entrevistada, las características de la asociación y su actividad, las relaciones de la entidad con las administraciones públicas y otros agentes sociales y la participación de mujeres en la asociación.

En los apartados que siguen se presentará, en primer lugar, un breve análisis del estado de la cuestión en lo que respecta a las corrientes teórico-analíticas en el estudio de la participación política de las comunidades migrantes, para pasar a exponer brevemente el contenido de cada una de las contribuciones que componen este número especial.

\section{La integración política de las comunidades migrantes: Entre el análisis sociodemográfico y la emergencia de la perspectiva transnacional}

La participación política de las comunidades migrantes en los países de destino y origen, entre los que tejen sus redes de vínculos y prácticas transnacionales, es un tema clave en el marco de los estudios migratorios, así como también en otros escenarios de estudio que, en el ámbito principalmente de la sociología y la ciencia política, abordan cuestiones tales como la ciudadanía, la participación de la comunidad civil en la vida pública o la integración y la cohesión social.

La relación entre procesos sociodemográficos y formas asociativas de las comunidades migrantes ha sido tradicionalmente uno de los ejes de investigación más productivos. Partiendo del ya clásico estudio de Rex et al. (1987) sobre las entidades de migrantes en Europa, las contribuciones que se orientan en torno a este eje han analizado la relación entre los flujos y los procesos migratorios y las características del asociacionismo migrante (Moya, 2005; Vermeulen, 2010; Voicu y Rusu, 2012; Bada, 2013), pero también cuestiones vinculadas al liderazgo en el seno de las comunidades (Pratten y Baldo, 1995; Escala et al., 2011; Antwi, 2010; Pirkkalainen et al., 2013) o la participación femenina en dichas organizaciones (Pojmann, 2007; Jettinger, 2010; De Tona, 2012). En el caso español existen también importantes contribuciones que se enmarcan en esta línea. Entre otras, encontramos: Veredas, 1998; Aparicio y Tornos, 2010; Garreta y Llevot, 2013; Giró y Mata, 2013; y en referencia a la cuestión de género: Ramírez, 1998; Molina et al., 2013.

Otra de las líneas más fructíferas en el análisis de la participación política de los inmigrantes es aquella que considera a las asociaciones de migrantes como un vehículo primordial del proceso de integración. Es ese caso se estudian cuestiones como las relaciones de los poderes públicos con las asociaciones de migrantes o la capacidad de estas entidades de generar recursos de apoyo para su comunidad (Veredas, 2003; Odmalm, 2004; Amelina y Faist, 2008; Strunk, 2014; Cebolla-Boado y López-Sala, 2015), partiendo bien de la perspectiva del capital social, bien de los análisis que enmarcan a estas aso- 
ciaciones en el paradigma de los nuevos movimientos sociales (Koff, 2005; Morales y Giugni, 2011).

La emergencia del paradigma transnacional en los estudios migratorios ha permitido, por una parte, dejar de lado el nacionalismo metodológico y, por otra, en el estudio de la temática que nos concierne, considerar las prácticas asociativas de las comunidades migrantes como parte de un proceso de transnacionalismo político que dialoga con los países de acogida (Caglar, 2006; Fitzgerald, 2008), pero que también tiene cada vez más relevancia para los países de origen. En este ámbito ha sido central la publicación del estudio de Levitt (2001), The Transnational Villagers.

Entre las temáticas abordadas por los exámenes que parten de la perspectiva del transnacionalismo político destacan aquellos que analizan la implicación de los estados con su diáspora organizada (Bakewell, 2006), tanto a través de la garantía de derechos - principalmente regulando el sufragio a residentes en el exterior (Itzigsohn y Villacrés, 2008; Laffleur y Calderón, 2011) - como implementando programas de desarrollo y codesarrollo que implican a las comunidades migrantes como actores en este proceso (Orozco, 2002; Lacroix, 2009; Østergaard-Nielsen, 2011; Lacomba y Escala, 2013: Morales y Pilati, 2014).

No obstante, el actual período de crisis, así como la introducción de medidas de corte austeritario para su gestión, han implicado un adelgazamiento de los fondos públicos destinados a políticas de integración de inmigrantes, pero también una reducción y una reestructuración de los organismos en los que participaban como agentes sociales las asociaciones de migrantes (Bueno y Treviño, 2015; Treviño y González-Ferrer, 2016). En este contexto es de esperar que cambie el enfoque prioritario de la academia en relación con la actividad política de las comunidades migrantes, si bien son todavía escasas las publicaciones que abordan el impacto de la crisis en los procesos y en las formas asociativas de estas comunidades. Cabe destacar aquí el análisis de Ejorh (2015) sobre la resiliencia en la adaptación de las entidades a una situación de crisis en el caso irlandés, o también la contribución de Cebolla-Boado y López-Sala (2015) sobre el activismo transnacional latinoamericano en España, así como el capítulo de Oliveri (2014), que aborda la reorientación de las luchas de las comunidades migrantes en Italia en el ámbito de la crisis.

En este contexto, se hacen necesarias nuevas contribuciones al conocimiento de activismo político transnacional de las comunidades migrantes en España que contribuyan a arrojar luz sobre algunas de las preguntas que se nos antojan de relieve, por ejemplo: ¿¿cuáles han sido los principales cambios de las formas asociativas de las comunidades migrantes en el período de crisis? ¿Han reorientado estas entidades sus actividades y demandas? ¿Cómo han variado las relaciones de las asociaciones de migrantes con otras entidades de la sociedad civil como las oenegés, pero también con las administraciones públicas? ¿Cuáles han sido los efectos de los procesos de retorno en las prácticas transnacionales de las asociaciones de migrantes como agentes de desarrollo pero también de integración? 


\section{Estructura del monográfico}

El monográfico se compone de siete artículos de investigación, tres de los cuales se corresponden con los resultados del proyecto de investigación Asociacionismo migrante en Galicia: Lazos transnacionales y codesarrollo desde una perspectiva de género y los otros cuatro son contribuciones originales sobre la participación política y asociativa de la población migrante en España.

El primer artículo, firmado por Vázquez-Silva, Pérez-Caramés y Mosquera Mirás, presenta un análisis de la evolución del tejido asociativo migrante en Galicia en la última década (2006-2016), marcada en su inicio por el boom migratorio y en su extremo por la dureza de la recesión. Bajo un ángulo sociodemográfico de análisis, se indaga en el cambio del perfil y del tamaño asociativo y se indaga en sus factores explicativos.

El segundo texto lleva por título «La representación política de las personas de origen inmigrante en España e Italia» y corre a cargo de Daniela Vintila y Laura Morales, quienes presentan un análisis de la presencia de migrantes en los partidos políticos españoles e italianos como cargos electos en los respectivos parlamentos. La contribución se enmarca en un estudio de las opiniones acerca de la participación política de las personas inmigrantes, en relación y en comparación con las posiciones de los diversos partidos de España e Italia en materia de política migratoria.

A continuación recogemos la contribución de Cortés Maisonave y Sanmartín Ortí en torno a la dimensión transnacional de la agencia asociativa migrante. El estudio se centra en las actividades vinculadas al codesarrollo por parte de diversas asociaciones de migrantes residentes en España y, más concretamente, en la ciudad de Madrid, además se analiza con detalle la relación que, en este ámbito de actividad, mantienen las asociaciones de migrantes con las oenegés dedicadas a la cooperación para el desarrollo.

En cuarto lugar, el artículo titulado "La participación política de las mujeres migrantes a través de las asociaciones en Galicia: Liderazgo y relaciones de poder», de Luzia Oca y Xabier Sánchez Lombardero, aborda las relaciones de género en las asociaciones de migrantes y propone una tipología asociativa que combina el carácter mixto o no de dichas entidades, así como la presencia o la ausencia de mujeres a la cabeza de estas organizaciones. El análisis se complementa con un estudio de caso de las comunidades migrantes, principalmente de origen caboverdiano y peruano, en la localidad gallega de Burela.

Moraes y Cutillas firman la contribución titulada «La estructura de oportunidad política transnacional y el giro relacional en el análisis de la participación política y el asociacionismo migrante», en la que realizan una crítica al enfoque de la estructura de oportunidades políticas (EOP) para la explicación de la participación política de los migrantes y las migrantes en España y proponen un marco analítico que, bajo un prisma transnacional, incorpore elementos relacionales y dinámicos al análisis de la involucración activista migrante.

Esther Cano-Ruiz y Belén Fernández Suárez presentan en su artículo un análisis de las redes relacionales de las entidades asociativas formadas por la 
población migrante gallega a partir de las teorías del capital social, concluyendo con la relevancia de la estructura de oportunidades que ofrecen los contextos locales, los más cercanos a la vida de las personas, para el desarrollo y la actividad de las organizaciones migrantes.

La contribución que cierra este monográfico corre a cargo de Fernández Suárez, Verdía Varela y De Palma Úngaro, quienes analizan los procesos de integración de la población migrante en Galicia desde un doble enfoque que considera, por una parte, la percepción que las personas migrantes tienen respecto al concepto y al proceso de integración y, por otra, el desarrollo de políticas a este respecto por parte de los municipios de mayor concentración de población migrada.

\section{Agradecimientos}

Queremos agradecer a la Dirección Xeral de Relacións Exteriores y coa Unión Europea (Xunta de Galicia) la concesión y la dotación económica del proyecto, así como a la ONGD Ecos do Sur su colaboración activa en esta investigación. Además, queremos mostrar un agradecimiento especial a todas aquellas personas que han colaborado en la confección de este estudio como informantes y que han compartido así un pedazo de sus vidas con nosotros, y también a quienes han participado en la transcripción de las entrevistas llevadas a cabo y en la realización del cuestionario telefónico a asociaciones de migrantes en Galicia.

\section{Referencias bibliográficas}

AmelinA, A. y FAIST, T. (2008). «Turkish Migrant Associations in Germany: Between Integration Pressures and Transnational Linkages». Revue Européenne des Migrations Internationales, 24(2), 91-120. $<$ https://doi.org/10.4000/remi.4542>

ANTWI, T. (2010). «Leadership and Membership Structure of Migrant Associations: The Case of Nigerian Migrant Associations in Accra, Ghana». Journal of Social Sciences and Public Policy, 2, 37-51.

Aparicio, R. y Tornos, A. (2010). Las asociaciones de inmigrantes en España: Una visión de conjunto. Madrid: Ministerio de Trabajo e Inmigración.

BADA, X. (2013). «From National to Topophilic Attachments: Continuities and Changes in Chicago's Mexican Migrant Organizations». Latino Studies, 11(1), 28-54. <https://doi.org/10.1057/lst.2012.53>

BAKEWELL, O. (2006). «Perspectives from Governments of Countries of Origin and Migrant Associations». Migración y Desarrollo, 7, 204-220.

Bueno, X. y TReviÑo, R. (2015). «Los municipios ante la disminución del Fondo para la Integración: Percepciones desde la gestión local». En: ARANGO, J.; MOYA, D.; Oliver, J. y SÁnchez-Montijano, E. (eds.). Anuario de la Inmigración en España: Flujos cambiantes, atonía institucional. Barcelona: CIDOB, 250-275.

Caglar, A. (2006). «Hometown Associations, the Rescaling of State Spatiality and Migrants Grassroots Transnationalism». Global Networks, 6(1), 1-22.

$<$ https://doi.org/10.1111/j.1471-0374.2006.00130.x> 
Cebolla-Boado, H. y López-Sala, A. (2015). «Transnational Latin American Immigrant Associations in Spain During the Economic Recession: A Top-Down Model of Integration and Transnationalism at Stake?». En: AYSA-LASTRA, M. y CACHÓN, L. (eds.). Immigrant Vulnerability and Resilience. Nueva York: Springer, 163-180.

De Tona, C. (2012). «Investing in Hope?: Gendered Resistance and the Struggle of Migrant Women's Associations in Ireland». En: LENTIN, R. y MoreO, E. (eds.). Migrant Activism and Integration from Below in Ireland. Nueva York: Springer, 95-108. <https://doi.org/10.1057/9780230369245_5>

EJORH, T. (2015). "The Challenge of Resilience: Migrant-Led Organisations and the Recession in Ireland». Journal of International Migration and Integration, 16(3), 679-699. <https://doi.org/10.1007/s12134-014-0361-5>

Escala, L.; Rivera-Salgado, G. y RodrígueZ, R. (2011). «Is More Necessarily Better? Leadership and Organizational Development of Migrant Hometown Associations in Los Angeles, California». Migraciones Internacionales, 6(2), 41-73.

FitzGerald, D. (2008). "Colonies of the Little Motherland: Membership, Space, and Time in Mexican Migrant Hometown Associations». Comparative Studies in Society and History, 50(1), 145-169. <https://doi.org/10.1017/S001041750800008X>

GARRETA, J. y LLEVOT, N. (2013). «Las asociaciones de inmigrantes africanos: Organización, proyección y actuaciones». Revista Internacional de Sociología, 71(1), 15-38. <https://doi.org/10.3989/ris.2012.09.01>

Giró, J. y MATA, A. (2013). «Las estructuras asociativas de los senegaleses en España». Revista Internacional de Sociología, 71(1), 91-115. <https://doi.org/10.3989/ris.2012.09.26>

ItZigsohn, J. y Villacrés, D. (2008). «Migrant Political Transnationalism and the Practice of Democracy: Dominican External Voting Rights and Salvadoran Home Town Associations». Ethnic and Racial Studies, 31(4), 664-686. <https://doi.org/10.1080/01419870701784497>

JetTinger, G.B. (2010). Unravelling Gender and Participation in Migrant Associations: An Ethnographic Study of a Senegalese Village Community in Paris, Dakar and Sinthiane. Tesis doctoral defendida en la Universidad de Oxford.

Koff, H. (2005). «Migrant Participation in Local European Democracies: Understanting Social Capital through Social Movement Analysis». Migraciones Internacionales, $3(2), 5-28$.

LACOMBA, J. y EsCALA, L. (2013). "Limits and challenges for the participation of migrants' associations in state development policies in Morocco and Mexico». International Review of Sociology, 23(1), 161-179. <https://doi.org/10.1080/03906701.2013.771044>

LACROIX, T. (2009). "Transnationalism and Development: The Example of Moroccan Migrant Networks». Journal of Ethnic and Migration Studies, 35(10), 1665-1678. <https://doi.org/10.1080/13691830903165865>

Laffleur, J.-M. y Calderón, L. (2011). "Assessing Emigrant Participation in Home Country Elections: The Case of Mexico's 2006 Presidential Election». International Migration, 49(3), 99-124. <https://doi.org/10.1111/j.1468-2435.2010.00682.x>

LeVITT, P. (2001). The Transnational Villagers. Berkeley: University of California Press. 
Molina, F.; SAmper, L. y Mayoral, D. (2013). «Liderazgo femenino: Un análisis de las diferencias de género en la formación y desarrollo de asociaciones de inmigrantes africanos». Revista Internacional de Sociología, 71(1), 141-166. <https://doi.org/10.3989/ris.2012.09.24>

Morales, L. y Giugni, M. (eds.) (2011). Social Capital, Political Participation and Migration in Europe: Making Multicultural Democracy Work? Londres: Palgrave Macmillan. <https://doi.org/10.1057/9780230302464>

Morales, L. y Pilati, K. (2014). "The Political Transnationalism of Ecuadorians in Barcelona, Madrid and Milan: The Role of Individual Resources, Organizational Engagement and the Political Context». Global Networks, 14(1), 80-102. <https://doi.org/10.1111/glob.12018>

MoYA, J.C. (2005). «Immigrants and Associations: A Global and Historical Perspective». Journal of Ethnic and Migration Studies, 31(5), 833-864. <https://doi.org/10.1080/13691830500178147>

Odmalm, P. (2004). «Civil Society, Migrant Organisations and Political Parties: Theoretical Linkages and Applications to the Swedish Context». Journal of Ethnic and Migration Studies, 30(3), 471-489. <https://doi.org/10.1080/13691830410001682043>

OliverI, F. (2014). «Acts of Citizenship against Neoliberalism: The New Cycle of Migrant Struggles in Italy». En: KIM, N.-K. (ed.). Multicultural Challenges and Sustainable Democracy in Europe and East Asia. Nueva York: Springer, 221-242.

Orozco, M. (2002). «Latino Hometown Associations as Agents of Development in Latin America». En: GarZA, R.O. de la y Lowell, B.L. (eds.). Sending Money Home: Hispanic Remittances and Community Development. Lanham, MD: Rowman \& Littlefield, 85-99.

ØstergaArd-Nielsen, E. (2011). «Codevelopment and Citizenship: The Nexus between Policies on Local Migrant Incorporation and Migrant Transnational Practices in Spain». Ethnic and Racial Studies, 34(1), 20-39. <https://doi.org/10.1080/01419871003777791>

Pirkkalainen, P.; Mezzeti, P. y Guglielmo, M. (2013). «Somali Associations’ Trajectories in Italy and Finland: Leaders Building Trust and Finding Legitimisation». Journal of Ethnic and Migration Studies, 39(8), 1261-1279. <https://doi.org/10.1080/1369183X.2013.778146>

Pojmann, W. (2007). «Organizing Women Migrants: The Philipino and Cape Verdean Women's Association's in Rome». Migration Letters, 4(1), 29-39.

Pratten, D. y Baldo, S.A. (1995). "Return to the roots: Processes of legitimacy in sudanese migrant associations». En: Edwards, M. y Hulme, D. (eds.). Non-Governmental Organisations - Performance and Accountability: Beyond the Magic Bullet. Londres: Earthscan, 119-129.

RAMíreZ, Á. (1998). "Relacionarse en Madrid: Mujeres, hombres y asociacionismo marroquí». Ofrim/Suplementos (diciembre), 103-124.

ReX, J.; Joly, D. y Wilpert, C. (1987). Immigrant associations in Europe. Gower: Aldershot.

STRUNK, C. (2014). "We are Always Thinking of our Community': Bolivian Hometown Associations, Networks of Reciprocity, and Indigeneity in Washington DC». Journal of Ethnic and Migration Studies, 40(11), 1697-1715. <https://doi.org/10.1080/1369183X.2013.871492> 
TreviÑo, R. y GONZÁLEZ-Ferrer, A. (2016). «Gestión local de la inmigración en los inicios de la crisis económica». En: DomingO, A. (ed.). Inmigración y diversidad en España: Crisis económica y gestión municipal. Barcelona: Icaria, 75-98.

VEREDAS, S. (1998). «Las asociaciones de inmigrantes marroquíes y peruanos en la Comunidad de Madrid». Universidad Complutense de Madrid. Tesis doctoral.

- (2003). «Las asociaciones de inmigrantes en España: Prácticas clientelares y cooptación política». Revista Internacional de Sociología, 61(36), 207-225. <https://doi.org/10.3989/ris.2003.i36.320>

Vermeulen, F. (2010). «Organisational Patterns: Surinamese and Turkish Associations in Amsterdam, 1960-1990». Journal of Ethnic and Migration Studies, 31(5), 951-973. <https://doi.org/10.1080/13691830500177859>

Voicu, M. y Rusu, I.A. (2012). "Immigrants' Membership in Civic Associations: Why are Some Immigrants more Active than Others?». International Sociology, 27(6), 788-806.

<https://doi.org/10.1177/0268580912452172> 